\title{
El principio cooperativo de educación, formación e información desde una perspectiva histórica y doctrinal ${ }^{1}$
}

(The cooperative principle of education, training and information from a historical and doctrinal perspective)

\author{
Alejandro Martínez Charterina² \\ Universidad de Deusto (España)
}

Sumario: 1. El quinto principio cooperativo y sus referencias históricas. 2. Análisis del principio. 3. Relación del principio con los valores cooperativos. 4. Inquietudes y planteamientos de futuro relativos a la educación cooperativa. 5. Bibliografía.

Summary: 1. The fifth cooperative principle and its historical references. 2. Analysis of the principle. 3. Relationship of the principle with the cooperative values. 4 . Concerns and future approaches relatives to cooperative education. 5. Bibliography.

Resumen: El principio de educación ha sido considerado razonablemente la «regla de oro» del cooperativismo. Constituye la puerta al conocimiento de los caracteres esenciales de la cooperativa y al ejercicio de los derechos y responsabilidades de los socios. Al mismo tiempo acerca la cooperativa como empresa a la sociedad, y muy especialmente a los jóvenes que pueden encontrar en ella un futuro satisfactorio, dando continuidad al movimiento cooperativo en el tiempo. rativos

Palabras clave: cooperativas, identidad cooperativa, principios coope-

Abstract: The principle of education has reasonably been considered the "golden standard» of cooperativism. It is the gateway to knowledge of the essential characteristics of the cooperative and to the exercise of the rights and responsabilities of the partners. At the same time it brings the co-

1 Texto basado en la ponencia del mismo título presentada en el Congreso de la Asociación Internacional de Derecho Cooperativo y la Academia Vasca de Derecho sobre «El principio de educación, formación e información de las cooperativas», en Bilbao, el 13 de noviembre de 2019.

2 Catedrático Emérito de la Universidad de Deusto. E-mail: amartin@deusto.es. 
operative as a company close to society, and specially to young people who can find in it a satisfactory future, continuing the cooperative movement through the time.

Keywords: cooperatives, cooperative identity, cooperative principles. 


\section{El quinto principio cooperativo y sus referencias históricas}

La educación ha constituido un elemento esencial e inseparable de la actividad empresarial cooperativa en todo momento.

Antes, incluso, de la Cooperativa de Rochdale, la que consideramos el origen del cooperativismo moderno, encontramos a los llamados precursores que concedieron una gran importancia a la educación en el marco de sus proyectos.

En el falansterio de Charles Fourier no sólo hay biblioteca y salas de estudio $^{3}$, sino que existe un programa de educación de autorrealización por etapas, crítico con el sistema educativo convencional4.

Del mismo modo, Robert Owen mostró su inquietud por la educación desde la infancia, con excelentes resultados en su obra NewLanark, en la que el programa educativo, que debía proporcionar el desarrollo físico y moral, se extendía hasta los 14 años en escuelas elementales y a partir de esa edad en escuelas politécnicas ${ }^{5}$.

Por su parte, William King, discípulo de Owen, creó en Brighton y su entorno escuelas cooperativas, en el contexto de las múltiples cooperativas de consumo que se fueron formando bajo su impulso, a las que asignaba una tarea de educación profunda6. Es de destacar también la publicación del periódico The Cooperator, que realizó, redactándolo personalmente, durante veintiocho meses, entre 1828 y $1830^{7}$.

Y también en otros precursores, como Louis Blanc, Peztalozzi, Fullenberg, Grundtvig, Fröbel, y varios más, está presente la importancia de la educación en sus obras ${ }^{8}$.

En los Estatutos Primitivos de la Cooperativa de Rochdale, de 1844, en su artículo primero se dice: «Desde el momento en que sea posible, esta sociedad emprenderá la organización de las fuerzas de la producción, de la distribución, de la educación y del gobierno, o, dicho en otras palabras, el establecimiento de una colonia que se baste a sí misma y en la que se unirán los intereses, o bien prestará ayuda a otras sociedades para establecer colonias de esta clase». Y añade a continuación: «Para desarrollar la sobriedad, se abrirá una Sala de Tem-

3 Gromoslav Mladenatz, Historia de las doctrinas cooperativas, (1969), p. 42

4 Puede verse Dominique Desanti, Los socialistas utópicos, (1973), ps. 213 ss.

5 Alicia Kaplan de Drimer y Bernardo Drimer, Las cooperativas: fundamentos, historia, doctrina, (1981), p. 206.

6 Paul Lambert, La doctrina cooperativa, (1970), p. 40.

7 Alicia Kaplan de Drimer y Bernardo Drimer, o.c., (1981), p. 212.

8 Puede verse Margot Hendges, El principio de la educación cooperativa: estudio y aplicación en la región del «Vale dos sinos» - Brasil, (2005), ps. 112-114. 
planza tan pronto como se crea conveniente, en una de las casas de la sociedad»?.

Situados en Manchester, en el segundo cuarto del siglo XIX, en el contexto de un proceso de industrialización que ha hecho aparecer la clase trabajadora y el «problema social» que viene asociado a este proceso, no habiendo podido los trabajadores conseguir un aumento de sus salarios a pesar de la buena situación económica de la que venían gozando las empresas, de entre los distintos movimientos político-sociales que van apareciendo, los veintiocho tejedores que van a promover la Cooperativa de Rochdale se van a enfrentar a la situación del comercio minorista de provisiones que les lleva a vivir endeudados con la tienda de su barrio y a soportar pesos y calidades inferiores a los deseados.

Para hacer comprender la situación a estas personas es imprescindible una acción educativa: «educar a la gente de escasos recursos antes de poderla servir», en expresión de Holyoake ${ }^{10}$.

"Los primeros cooperativistas vivían en sociedades en las que la educación estaba reservada a los privilegiados», y como sucede en la actualidad, se dieron cuenta de la necesidad de alcanzar un grado educativo que facilitara una vida mejor ${ }^{11}$.

El almacén se instala en el bajo de una casa en la calle Tood Lane de Rochdale, que alquilan por tres años. El edificio tenía tres pisos y buhardilla. Poco tiempo después, en 1848, alquilaron el edifico entero por 21 años más.

El segundo piso se convirtió en sala de reuniones y fue provista de diarios para el uso de los socios. Pronto se estableció una sección de venta de libros y diarios, y los beneficios de esta sección se destinaban a la adquisición de libros para la sociedad ${ }^{12}$.

En 1850 se puso en marcha una escuela para niños, y pocos años después se amplió con una nueva aula destinada a personas entre 14 y 40 años $^{13}$.

9 «That as son as practicable, this society shall proceed to arrange the powers of production, distribution, education, and government, or in other words to stablish a self-supporting home-colony of united interests, or assist other societies in establishing such colonies», y "That for the promotion of sobriety a Temperance Hotel be opened in one of the society's houses, as son as convenient». Laws and objects of the Rochdale Society of Equitable Pioneers (1844), p. 3.

10 Georges Jacob Holyoake, Historia de los Pioneros de Rochdale, (1989), p. 19

11 Alianza Cooperativa Internacional, Notas de orientación para los principios cooperativos, (2015), p. 63.

12 Georges Jacob Holyoake, o.c., (1989), p. 28.

13 Georges Jacob Holyoake, O.C., (1989), p. 88. 
Diez años después de la constitución de la Sociedad, en los Estatutos que adopta la Asamblea General de 23 de octubre de 1854, se establece la constitución de un fondo para el perfeccionamiento intelectual de los socios y sus familias, para el mantenimiento de la biblioteca que iba creciendo en esos años, y para otros medios de instrucción que fueran adoptados. El fondo se constituye con el 2,5\% de los beneficios y por el importe acumulado de las multas por infracción de los Estatutos, y su gestión se realizará por una comisión de 11 miembros $^{14}$.

Unos años antes, en 1849, la Sociedad había organizado la recogida de donativos de dinero y libros para la Biblioteca y había constituido un comité directivo para su gestión, poniendo en marcha, de esa forma, lo que después se denominó Departamento de Educación ${ }^{15}$.

Con todo ello, como pone de manifiesto Holyoake, a través de la educación, a la que denomina «regla de oro», se pretendió contribuir al progreso moral e intelectual de los miembros de la cooperativa ${ }^{16}$.

De este modo se establece en la cooperativa de Rochdale, junto a las demás reglas aplicables en la misma, la de promover la educación entre sus miembros, tratando éstos pioneros de elevar a las personas a un nivel moral más elevado a través del cooperativismo ${ }^{17}$.

A partir de la creación de la Alianza Cooperativa Internacional, en 1895, será ésta organización la que se ocupará de la custodia y actualización de los principios cooperativos ${ }^{18}$.

Si durante bastantes años no hubo una acción directa sobre el tema, en el X Congreso de la Alianza, celebrado en Basilea en 1921, mediante una resolución se reconocieron los principios de Rochdale, pidiendo a las cooperativas afiliadas a las organizaciones miembros que sus comportamientos se adecuaran a los mismos ${ }^{19}$.

Desde entonces la Alianza ha revisado en tres ocasiones los principios para acomodarlos, hasta desembocar en la Declaración de la identidad cooperativa, en su centenario.

La primera de las revisiones se produjo en el XV Congreso que tuvo lugar en París en 1937. En esta revisión se aprobó el informe del Comité especial que se constituyó para ello, quedando los siete principios

14 Paul Lambert, o.c., (1970), p. 334.

15 G.J. Holyoake, o.c. (1989), p. 87.

16 G.J. Holyoake, o.c. (1989), p. 93.

17 Paul Lambert, O.C., (1970), p. 87.

18 En el artículo 1 del Reglamento de la Alianza Cooperativa Internacional, de 11 de abril de 2013 (modificado el 13 de noviembre de 2015 y el 17 de noviembre de 2017), se dice «la $\mathrm{ACl}$ es la guardiana de los valores y principios cooperativos».

19 José Odelso Schneider, Democracia, participaçao e autonomía cooperativa, (1991), p. 49. 
cooperativos divididos en dos categorías, los cuatro primeros necesarios para la afiliación de una cooperativa a la Alianza, y los tres últimos recomendables, aunque no necesarios. El tercero de estos era precisamente la promoción de la educación.

El Comité concluía que debía mantenerse la promoción de la educación como principio, sin determinar qué recursos habían de comprometer para ello, lo que podía variar según las circunstancias, si bien aconsejando dedicar una parte de los excedentes netos de la cooperativa a tal fin ${ }^{20}$.

La segunda revisión se encargó en el XXII Congreso de la Alianza a una Comisión para el estudio de los principios, en Bournemouth en 1963, y la reforma se aprobó en el XXIII Congreso, que se celebró en Viena en 1966.

Los principios eran seis, todos ellos necesarios, y el quinto de ellos era el de educación, redactado en estos términos:

«Todas las sociedades cooperativas deben tomar medidas para promover la educación de sus miembros, dirigentes, empleados y público en general, en los principios y métodos de la cooperación, desde el punto de vista económico y democrático» ${ }^{21}$.

El Informe realizado a la Alianza para la reforma de los principios en 1966 entiende que la educación debe ser norma fundamental, considerando que este principio «hace posible la efectiva vigencia de los demás principios cooperativos», toda vez que «el cooperativismo no se funda en los impulsos egoístas del hombre ni en la ciega obediencia, sino en la autodisciplina colectiva, y requiere por lo tanto la adopción de nuevas ideas y nuevas normas de conducta que solo pueden adquirirse por una apropiada educación $»^{22}$.

Al mismo tiempo el principio de educación extiende ésta a los miembros de la cooperativa, para facilitar una realización correcta de sus derechos y obligaciones como socios, a los dirigentes, ya sean socios elegidos o administradores contratados, para el correcto desenvolvimiento de la gestión de la empresa, y, además de dirigirse al interior, el principio se proyecta al exterior, al dirigirse al público en general, al que se desea informar acerca de la cooperativa y sus actividades, entendiendo que ésta se sitúa en un entorno social con el que debe relacionarse e interactuar ${ }^{23}$.

20 Alicia Kaplan de Drimer y Bernardo Drimer, o.c., (1981), p. 565.

21 Tomado de Alicia Kaplan de Drimer y Bernardo Drimer, o.c., (1981), p. 565.

22 Alicia Kaplan de Drimer y Bernardo Drimer, o.c., (1981), p. 565.

23 En este sentido, Alicia Kaplan de Drimer y Bernardo Drimer, o.c., (1981), p. 566. 
En el Congreso centenario de la Alianza Cooperativa Internacional, celebrado en Manchester en 1995, se aprobará la Declaración de la Identidad Cooperativa que supone la última de las revisiones de los principios cooperativos, así como la introducción de los valores cooperativos conformando esta identidad. Entre los principios, de nuevo en quinto lugar, el de educación, bajo el nombre de «educación, formación e información».

El enunciado del principio en el texto de la Declaración es el siguiente: «Las cooperativas proporcionan educación y formación a los socios, a los representantes elegidos, a los directivos y a los empleados para que puedan contribuir de forma eficaz al desarrollo de sus cooperativas. Informan al gran público, especialmente a los jóvenes y a los líderes de opinión, de la naturaleza y beneficios de la cooperación ${ }^{24}$.

Además de estos tres Congresos en los que se establecen actualizaciones de los principios, son muchos los que se han ocupado del principio de educación ${ }^{25}$, referidos en orden cronológico y sin ánimo de ser exhaustivo:

- El I Congreso de Londres, 1895. En la resolución 11 del mismo se considera que para promover la educación social de las personas las cooperativas podrían establecer fundaciones educativas, secciones de mujeres y otras instituciones auxiliares.

- El II Congreso de París, 1896. En este Congreso hay propuestas para que la cooperación se considere materia de enseñanza y se promocione la educación de adultos.

— El IV Congreso de París, 1900. Una resolución invita a que las cooperativas de todos los países promocionen la educación de sus miembros.

—El IX Congreso de Glasgow, 1913. Se recomienda en esta Congreso que las Uniones Cooperativas publiquen periódicos de forma regular como instrumento de educación de los miembros de las cooperativas y de sus familias.

—El X Congreso de Basilea, 1921. El informe del Presidente de la Alianza dedica una parte a la educación, la importancia de las lenguas, de la propaganda y la información. Se felicita la creación de la Cátedra de Cooperativismo en la Universidad de París, y se establece la celebración del Día de la Cooperación.

24 I.C.A.: Declaración de la Alianza Cooperativa Internacional sobre la Identidad Cooperativa, (1996), p. 19.

25 Ampliamente estudiado en Margot Hendges, O.C., (2005), ps. 140-170. 
- El XII Congreso de Estocolmo, 1927. Se trataron temas acerca de la información entre movimientos cooperativos de los distintos países en el campo de la educación profesional cooperativa, y a tales efectos la publicación de la Alianza, el Boletín, podría facilitar intercambios de información en dicha materia.

- El XIII Congreso de Viena, 1930. En este Congreso se planteó el estado de aplicación de los principios de Rochdale en los distintos países y se nombró un Comité preparatorio de las recomendaciones para la actualización de los principios del Congreso de 1937.

—EI XVIII Congreso de Copenhague, 1951. Una resolución plantea la dificultad para afrontar la problemática de su tiempo sin la educación precisa y pide, en consecuencia, contacto con los educadores para utilizar su conocimiento y técnicas en favor de la educación cooperativa. Considera la importancia de los gremios de mujeres en la educación organizada de los niños. Pide utilizar la prensa cooperativa, y supervisar la educación del personal de las cooperativas.

- El XXII Congreso de Bournemouth, 1963. De nuevo se creó una Comisión para el estudio de los principios cooperativos y su reforma, cuyo informe se llevó al siguiente Congreso en el que se actualizaron dichos principios. En este Congreso se trató también de la importancia del Centro Educativo del sudeste asiático de la Alianza para reforzar la educación cooperativa a través de las organizaciones cooperativas, los gobiernos y las Universidades, en esta región.

- El XXIV Congreso de Hamburgo, 1969. En este Congreso se presentó un informe acerca de la educación de los miembros de las cooperativas, considerando la educación como un proceso que dura toda la vida, y destacando los métodos para llevarla a cabo.

- El XXV Congreso de Varsovia, 1972. Al igual que en el anterior se presentó informe sobre la educación del periodo 69-72. En este periodo la $\mathrm{ACI}$ intensificó su relación con la UNESCO, de modo que en la Conferencia General de esta última se aprobó una resolución en la que se reconocen las ventajas del movimiento cooperativo para la educación de adultos, especialmente en países en vías de desarrollo. También se produjo el reconocimiento de la Alianza de la importancia de la educación desde sus orígenes para el desarrollo del movimiento cooperativo.

- El XXVI Congreso de París, 1976. En el Informe sobre el trabajo que el Comité Central de la Alianza realizó en materia de educación en el periodo inmediatamente anterior, se presenta la cons- 
titución del Cuerpo Consultivo de Educación en 1973, que en sus dos años de duración realizó el proyecto CET, una tarea de preparación de los materiales disponibles para la educación en los países en vías de desarrollo. También se llevó a cabo el proyecto CEMAS, servicio consultivo de materiales para la educación cooperativa.

- El XXVII Congreso de Moscú, 1980. El Informe que presentó Alex F. Laidlaw titulado "Las cooperativas en el año 2000», dedicó también su atención a la educación y, aunque no hubo una resolución sobre ella, se aprobó una moción para el uso de los medios de comunicación masivos en el movimiento cooperativo.

- El XXVIII Congreso de Hamburgo, 1984. Continuando en la línea del Informe Laidlaw, se da importancia a la educación y se destaca la necesidad de trasladar a los jóvenes la información de la presencia del cooperativismo en la sociedad.

- EI XXIX Congreso de Estocolmo, 1988. Se trata el tema de los valores básicos del cooperativismo. Entre los que se presentan están los vinculados a la educación: conocimiento, entendimiento, intuición. Se apoyó también la propuesta de la UNESCO para la proclamación de un año contra el analfabetismo.

- El XXX Congreso de Tokio, 1992. Tras los Congresos de Moscú, que identificó los problemas del futuro, de Hamburgo, que trató las perspectivas, y de Estocolmo, que profundizó en ellas, quedó para Tokio la preparación de lo que se llevaría al Congreso Centenario de Manchester de 1995, en el que se aprobaría la Declaración de la Identidad Cooperativa, a la que nos hemos referido, y, en consecuencia, el principio de educación, formación, e información, así como los valores cooperativos que constituyen sus aspiraciones.

\section{Análisis del principio}

El texto del principio en la Declaración de Identidad Cooperativa, que hemos transcrito anteriormente, refiere que las cooperativas proporcionan educación y formación a las personas que se encuentran dentro de la cooperativa como socios, representantes elegidos, directivos y empleados, pero también a las que están fuera, es decir a la sociedad en general, al gran público, en especial, a los jóvenes y a los líderes de opinión. 
Con relación a los primeros, la educación les proporciona «el conocimiento de los fundamentos del cooperativismo, el sentido mismo, los valores y principios a los que se acomoda la actividad cooperativizada, los derechos y responsabilidades. Es la forma en que unos y otros pueden entenderse en el marco de la cooperativa, pueden comprender las necesidades de los otros y el sentido de su aportación» ${ }^{26}$.

Los representantes elegidos, al igual que los directivos y empleados, han de estar bien formados disponiendo del conocimiento profesional necesario para el buen desempeño de sus funciones: "La formación significa asegurar que todos los que están implicados en las cooperativas tengan las habilidades necesarias para llevar a cabo sus responsabilidades eficazmente» ${ }^{27}$. En el pasado en muchos lugares tuvo gran importancia la presencia de administradores competentes en las cooperativas, que permitieron crecer a muchas de ellas en los fuertes entornos competitivos de los grandes mercados dejando atrás las pequeñas dimensiones locales que tenían.

Y, del mismo modo, las cooperativas, insertas en la sociedad, deben transmitir a ésta sus aportaciones y, tomando en consideración que la gestión democrática y social de la cooperativa puede resultar atractiva para ese cuerpo social, deben informar de los beneficios de la cooperación, especialmente a los jóvenes que se pueden sentir atraídos por «la diferencia cooperativa», en la consideración de Birchall28, así como a los líderes de opinión, verdaderos altavoces de movilización social29.

Por cuanto se refiere a los miembros de la cooperativa, la educación les llevará al conocimiento de los valores y principios cooperativos, así como a la forma de aplicarlos en el funcionamiento diario de la cooperativa, logrando, de este modo, cooperativistas más comprometidos.

Además, por extensión, la educación les conducirá a ser ciudadanos más activos, en la medida en que se proyecta en el desarrollo social de los miembros. En este sentido llamó Owen llamó a su primera escuela «Instituto para la formación del carácter»30.

La formación les permitirá tanto a los miembros representantes elegidos, como a los directivos y empleados desarrollar aptitudes prácticas que les permitan dirigir la cooperativa de forma ética y responsable ${ }^{31}$.

26 Alejandro Martínez Charterina, La cooperativa y su identidad, (2016), p. 69.

27 I.C.A.: Declaración..., o.c., (1996), p. 61.

28 Johnston Birchall, «Co-operative principles ten years on», (2005), p. 57.

29 Alejandro Martínez Charterina, o.c., (2016), p. 70.

30 A.C.I., Notas..., O.C., (2015), p. 67.

31 A.C.I., Notas..., O.C., (2015), ps. 65-66. 
La información al público trata de dar conocimiento de la empresa cooperativa, una empresa gestionada democráticamente a través de sus valores y principios, sabiendo que no se va a apreciar ni apoyar aquello que no se entiende. Destinatarios de esta información los jóvenes que se enfrentan a un mundo más duro y menos igualitario, resultado de la crisis financiera pasada, así como de la crisis presente y futura consecuencia del fenómeno de la pandemia del coronavirus COVID-19 a escala mundial.

Y junto a los jóvenes, esta información a la sociedad se dirige especialmente a los líderes de opinión: «todos aquellos que influyen en la opinión pública, tales como políticos, funcionarios, agentes de los medios de comunicación y educadores»32.

En este sentido han tenido gran importancia las informaciones del Año Internacional de las Cooperativas 2012, así como los diversos informes de Naciones Unidas y de la Organización Internacional del Trabajo sobre las cooperativas y su importancia cara a un futuro mejor.

\section{Relación del principio con los valores cooperativos}

El principio de educación está especialmente relacionado con los valores cooperativos de autoayuda y autorresponsabilidad.

El Informe que acompaña al texto de la Declaración sobre la Identidad Cooperativa de la A.C.I. indica que la autoayuda, como valor cooperativo, está basada en la creencia de que cada persona es capaz de dirigir y controlar su propio destino. En la cooperativa la autoayuda se ejercita de modo conjunto con los demás socios de la misma a través de la acción colectiva y la mutua responsabilidad. Ahora bien, a través de la educación las personas, cada una, progresan en su propio desarrollo, mejorando sus capacidades como socio y como persona.

Al mismo tiempo, la educación ha de ayudar notablemente a que los socios sean capaces de asumir la responsabilidad del funcionamiento de su cooperativa, de su promoción en la sociedad, y del mantenimiento de la independencia de la misma de cualquier otra organización pública y privada33.

Pero se debe señalar que la educación es la puerta de la comprensión de todos los valores y principios que conforman la identidad

32 A.C.I., Notas..., o.c., (2015), p. 65.

33 Puede verse I.C.A, Declaración..., o.C., (1996), p. 37; A.C.I., Notas..., O.C., (2015), p. 66; Alejandro Martínez Charterina, o.c., (2016), p. 90. 
cooperativa. Es por lo que se ha venido llamando al principio de educación la «regla de oro» de la cooperativa, o por lo que se califica a la educación como energía vital y motor de desarrollo cooperativo ${ }^{34}$.

\section{Inquietudes y planteamientos de futuro relativos a la educación cooperativa}

Estamos estudiando la educación como motor del desarrollo cooperativo en la consideración de que la educación, a través de la actividad de las personas, eleva el nivel de vida de la sociedad. Los movimientos cooperativos nacionales se esforzaron mucho en el pasado para proporcionar educación de las más diversas formas: editaron periódicos, revistas y libros; fueron pioneros en temas tales como la educación de adultos, el aprendizaje a distancia, y la idea de aprendizaje para toda la vida; formaron bibliotecas, pusieron en marcha colegios cooperativos. El resultado fue el progreso de las cooperativas en muchos países 35 .

La educación debe seguir siendo en el futuro ese motor de desarrollo cooperativo: "la educación en las cooperativas debe seguir siendo igual de atrevida, innovadora e imaginativa» como lo fue en el pasado $^{36}$.

Naturalmente las nuevas tecnologías ofrecen hoy unas posibilidades que deben aprovecharse para ello.

La Alianza Cooperativa Internacional propone entre los diversos temas relacionados con la educación a considerar en el futuro los siguientes ${ }^{37}$ :

- El aprovechamiento del legado cooperativo, como fuente de información ejemplar para las cooperativas de hoy ${ }^{38}$.

- La educación cooperativa en los estudios primarios, secundarios, universitarios y de postgrado.

- El apoyo a la educación en las economías emergentes, especialmente en África y otras economías en desarrollo, como apoyo a la consecución de los objetivos de desarrollo sostenible de Naciones Unidas.

\footnotetext{
34 A.C.I., Notas..., O.C., (2015), p. 63.

35 I.C.A.: Declaración..., O.C., (1996), ps. 79-81.

36 A.C.I., Notas..., O.C., (2015), p. 64.

37 A.C.I., Notas..., O.c., (2015), ps. 73-76.

38 Puede verse el sitio web Stories.coop, a modo de ejemplo.
} 
- La importancia de creación de Instituciones de educación superior de enseñanza cooperativa.

- La investigación académica y la colaboración en la misma.

- La mejora en la comprensión de la sociedad del significado de la empresa cooperativa, facilitando datos, estadísticas de empleo, resultado de la acción cooperativa.

Finalizo con la consideración de que la acción responsable del socio en su cooperativa, así como el reconocimiento de la aportación de las cooperativas en la sociedad se consigue mediante el ejercicio constante del principio de educación.

\section{Bibliografía}

ALIANZA COOPERATIVA INTERNACIONAL. 2015. Notas de orientación para los principios cooperativos, www.aciamericas.coop/IMG/pdf/guidance_notes_es.pdf

BIRCHALL, Johnston. 2005. "Co-operative principles ten years on», en Review of International Co-operation, Vol. 98, N. ${ }^{\circ}$ 2/2005. I.C.A., Geneva, ps. 4563.

DESANTI, Dominique. 1973. Los socialistas utópicos, Editorial Anagrama, Barcelona.

INTERNATIONAL CO-OPERATIVE ALLIANCE. 1996. Declaración de la Alianza Cooperativa Internacional sobre la Identidad Cooperativa. Los principios cooperativos, Consejo Superior de Cooperativas de Euskadi, VitoriaGasteiz.

HENDGES, Margot. 2005. El principio de la educación cooperativa: estudio y aplicación en la región del «Vale dos Sinos» - Brasil, Tesis Doctoral, Universidad de Deusto, Bilbao.

HOLYOAKE, Georges Jacob. 1989. Historia de los Pioneros de Rochdale, Intercoop, Buenos Aires.

KAPLAN DE DRIMER, Alicia y DRIMER, Bernardo. 1981. Las cooperativas: fundamentos, historia, doctrina, 3. ${ }^{\mathrm{a}}$ ed., Intercoop, Buenos Aires.

LAMBERT, Paul. 1970. La doctrina cooperativa, 3. ${ }^{a}$ ed., Intercoop, Buenos Aires.

Laws and objects of the Rochdale Society of Equitable Pioneers. 1844. En https:// www.rochdalepioneersmuseum.coop/wp-content/uploads/2014/08/REPSLaws-and-Objects-1844.pdf

MARTÍNEZ CHARTERINA, Alejandro. 2016. La cooperativa y su identidad, Dykinson, Madrid.

MLADENATZ, Gromoslav. 1969. Historia de las doctrinas cooperativas, Intercoop, Buenos Aires.

SCHEIDER, José Odelso. 1991. Democracia, participaçao e autonomía cooperativa, UNISINOS, Sao Leopoldo. 


\section{Derechos de autor}

El Boletín de la Asociación Internacional de Derecho Cooperativo es una revista de acceso abierto lo que significa que es de libre acceso en su integridad inmediatamente después de la publicación de cada número. Se permite su lectura, la búsqueda, descarga, distribución y reutilización legal en cualquier tipo de soporte sólo para fines no comerciales y según lo previsto por la ley; sin la previa autorización de la Editorial (Universidad de Deusto) o el autor, siempre que la obra original sea debidamente citada (número, año, páginas y DOI si procede) y cualquier cambio en el original esté claramente indicado.

\section{Copyright}

The International Association of Cooperative Law Journal is an Open Access journal which means that it is free for full and immediate access, reading, search, download, distribution, and lawful reuse in any medium only for non-commercial purposes, without prior permission from the Publisher or the author; provided the original work is properly cited and any changes to the original are clearly indicated. 